\title{
REFERENCES
}

1. S. Arima, Commutative group varieties, J. Math. Soc. Japan vol. 12 (1960) pp. 227-237.

2. A. Borel, Groupes linéaires algébriques, Ann. of Math. vol. 64(1956) pp. 20-82.

3. S. Lang, Abelian varieties, New York, Interscience, 1959.

4. M. Rosenlicht, Some basic theorems on algebraic groups, Amer. J. Math. vol. 78 (1956) pp. 401-443.

5. - Some rationality questions on algebraic groups, Ann. Mat. Pura Appl. vol. 43 (1957) pp. 25-50.

6. - A universal mapping property of generalized jacobian varieties, Ann. of Math. vol. 66 (1957) pp. 80-88.

7. A. Weil, Variêtês abéliennes et courbes algébriques, Paris, Hermann, 1948.

University of Califoria, Berkeley

\section{EXTENDING CHARACTERS ON SEMIGROUPS}

\section{KENNETH A. ROSS ${ }^{1}$}

W. W. Comfort has proved [1, Theorem 4.2] a theorem on approximating certain semicharacters on commutative semigroups. He used the structure theory established in [2] and expressed doubt as to the necessity of one of his hypotheses, namely core $S(\chi) \neq \Lambda$. His result suggested the following theorem, which tells us when a character on a subsemigroup of a commutative semigroup $G$ can be extended to a character on $G$. Because of its technical nature we will not state Comfort's theorem but we will state as a corollary to our theorem a result which implies his theorem directly (with the hypothesis core $S(\chi) \neq \Lambda$ dropped).

A bounded complex-valued function $\psi$ on a semigroup $G$ is called a semicharacter of $G$ if $\psi(x) \neq 0$ for some $x \in G$ and $\psi(x y)=\psi(x) \psi(y)$ for all $x, y \in G$. A character $\psi$ is a semicharacter for which $|\psi(x)|=1$ for all $x \in G$. We note that it follows from the theorem in [3] that any character can be extended to a semicharacter.

TheOREM. Let $G$ be a commutative semigroup and let $S \subseteq G$ be a subsemigroup. A character $\psi$ on $S$ can be extended to a character on $G$ if and only if $\psi$ satisfies:

$$
a, b \in S, x \in G, \text { and } a x=b x \text { imply } \psi(a)=\psi(b) .
$$

Received by the editors June 20, 1960 and, in revised form, October 27, 1960.

${ }^{1}$ Supported by a National Science Foundation pre-doctoral fellowship. 
Proof. The necessity of $\left({ }^{*}\right)$ is clear. For the sufficiency, we may suppose that $G$ has a unit. By Zorn's lemma, it suffices to choose an $x_{0} \in G-S$ and extend $\psi$ to a character $\psi_{0}$ on $S_{0}=\left\{s x_{0}^{\boldsymbol{k}}: s \in S, k \geqq 0\right\}$ that satisfies $\left({ }^{*}\right)$ on $S_{0}$. Three cases must be considered. We omit the details which are similar to those in [3] but we note that in each case the crucial matters to be checked are that $\psi_{0}$ is well-defined and that $\psi_{0}$ satisfies $\left(^{*}\right)$ on $S_{0}$. In Cases 2 and 3 , the denials of the previous cases are essential.

CASE 1. Suppose there exist $a_{0}, b_{0} \in S, x_{0} \in G-S$, and $y_{0} \in G$ such that $a_{0} x_{0} y_{0}=b_{0} y_{0}$. Then extend $\psi$ to $\psi_{0}$ such that $\psi_{0}\left(x_{0}\right)=\psi\left(b_{0}\right) / \psi\left(a_{0}\right)$.

CAse 2. Suppose Case 1 does not apply but that for some $x_{0} \in G-S$ and some $k_{1} \geqq 2$, we have $x_{0}^{k_{1}} \in S$. Then let $k_{0}$ be the least positive integer such that $x_{0}^{k_{0}} \in S$ and extend $\psi$ to $\psi_{0}$ such that $\psi_{0}\left(x_{0}\right)$ is any $k_{0}$ th root of $\psi\left(x_{0}^{k_{0}}\right)$.

CAse 3. Suppose Cases 1 and 2 do not apply. Then choose $x_{0} \in G-S$ arbitrarily and extend $\psi$ to $\psi_{0}$ so that $\psi_{0}\left(x_{0}\right)=1$.

We now state the corollary implying [1, Theorem 4.2].

COROLlaRY. Let $\chi$ be a semicharacter on a commutative semigroup such that $\chi(x)=0$ or $|\chi(x)|=1$ for all $x \in G$. Let $S(\chi)=\{x \in G:|\chi(x)|=1\}$ and suppose that $A$ is a subsemigroup of $G$ such that

(1) $S(\chi) \subseteq A$;

(2) $x \in G, y \in G-A$ imply $x y \in G-A$;

(3) $x, y \in S(\chi), z \in A$, and $x z=y z$ imply $\chi(x)=\chi(y)$.

Then there is a semicharacter $\psi$ on $G$ such that $\{x \in G:|\psi(x)|=1\}$ $=A$ and $\chi(x)=\psi(x)$ for $x \in S(\chi)$.

Proof. Let $\chi_{0}$ be $\chi$ restricted to $S(\chi)$ and extend to a character $\psi_{0}$ on $A$ using the preceding theorem. Then define $\psi(x)=\psi_{0}(x)$ for $x \in A$ and $\psi(x)=0$ for $x \in G-A$.

Note. The above theorem and the theorem of [3] lead one to ask what conditions are necessary to extend semicharacters that never take the value zero. A natural conjecture would be condition (*) above and condition (A) of [3]:

$$
a, b \in S, x \in G \text {, and } a x=b \text { imply }|\psi(a)| \geqq|\psi(b)| \text {. }
$$

However, consider the following example. ${ }^{2}$ Let $G$ be the commutative semigroup generated by $\left\{a_{1}, a_{2}, \cdots, b_{1}, b_{2}, \cdots, c, d\right\}$ and satisfying the relations:

$$
a_{1}^{k_{1}} a_{2}^{k_{2}} \cdots b_{1}^{m_{1}} b_{2}^{m_{2}} \cdots c^{p} d^{q}=a_{1}^{k_{1^{\prime}}{ }^{k_{2_{2}^{\prime}}}} a_{2} \cdots b_{1}^{m_{1^{\prime}}} b_{2}^{m_{2^{\prime}}} \cdots c^{p^{\prime}} d^{q^{\prime}},
$$

2 Dr. Comfort suggested this simplification of the author's original example. 
whenever $q>0, q^{\prime}>0, k_{n}-m_{n}=k_{n}^{\prime}-m_{n}^{\prime}$ for all $n, q-p=q^{\prime}-p^{\prime}$, and $q+\sum_{n=1}^{\infty} m_{n}=q^{\prime}+\sum_{n=1}^{\infty} m_{n}^{\prime}$. (In the expression (**) all but finitely many of the exponents are zero.) Let $S$ be the subsemigroup of $G$ generated by $\left\{a_{1}, a_{2}, \cdots, c\right\}$ and define $\psi$ on $S$ by

$$
\psi\left(a_{1}^{k_{1}} a_{2}^{k_{2}} \cdots c^{p}\right)=\prod_{n=1}^{\infty}\left(\frac{1}{n}\right)^{k_{n}} .
$$

Then

(i) $\psi$ never takes the value zero on $S$;

(ii) $a, b \in S, x \in G$, and $a x=b x$ imply $\psi(a)=\psi(b)$;

(iii) $a, b \in S, x, y \in G$, and $a x y=b y$ imply $|\psi(a)| \geqq|\psi(b)|$;

(iv) any extension of $\psi$ to a semicharacter on $G$ takes on the value zero.

Indeed, if $\psi_{0}$ extends $\psi$, then $\psi_{0}(d)=0$ since $a_{n} b_{n} d=c d^{2}$ implies that $\left|\psi_{0}(d)\right| \leqq 1 / n$ for all $n$. The example can be considerably simplified if only condition (A) is desired rather than condition (iii).

\section{REFERENCES}

1. W. W. Comfort, The isolated points in the dual of a commutative semi-group, Proc. Amer. Math. Soc. vol. 11 (1960) pp. 227-233.

2. E. Hewitt and H. S. Zuckerman, The $l_{1}$-algebra of a commutative semigroup, Trans. Amer. Math. Soc. vol. 83 (1956) pp. 70-97.

3. K. A. Ross, $A$ note on extending semicharacters on semigroups, Proc. Amer. Math. Soc. vol. 10 (1959) pp. 579-583.

UNIVERSITY OF WASHINGTON 